\title{
Integer points of analytic functions in a half-plane
}

\author{
Alastair N. Fletcher and J.K. Langley * \\ January 6, 2009
}

\begin{abstract}
It is shown that if $f$ is an analytic function of sufficiently small exponential type in the right half-plane, which takes integer values on a subset of the positive integers having positive lower density, then $f$ is a polynomial. MSC2000: 30D20, 30D35.
\end{abstract}

\section{Introduction}

A classical theorem of Pólya (see [14] and [21, p.55]) shows that $2^{z}$ is the slowest growing transcendental entire function which takes integer values at the non-negative integers. That is, let $f$ be entire and take integer values on $\mathbb{N} \cup\{0\}$. Pólya shows that if

$$
\limsup _{r \rightarrow \infty} \frac{M(r, f)}{2^{r}}<1, \quad \text { where } \quad M(r, f)=\max \{|f(z)|:|z|=r\},
$$

then $f$ is a polynomial and, further, that if

$$
M(r, f)=O\left(r^{N} 2^{r}\right)
$$

as $r \rightarrow \infty$ for some $N>0$, then there exist polynomials $P_{1}$ and $P_{2}$ such that $f(z) \equiv P_{1}(z) 2^{z}+P_{2}(z)$. Further results on integer-valued entire functions may be found in $[1,2,4,6,11,12,16,17,18,19,20]$.

*Both authors were supported by Engineering and Physical Sciences Research Council grant EP/D065321/1. 
This article is concerned with similar results for analytic functions in a half-plane. It was proved in [8, Lemma 5] that if $f$ is analytic of polynomial growth in the right half-plane and takes integer values at the positive integers, then $f$ is a polynomial. This result has several applications to value distribution theory and differential equations $[8,9,10]$. In [13], an analogue of Pólya's result for a half-plane is given. That is, let $f$ be analytic in the closed right half-plane $\Omega=\{z \in \mathbb{C}: \operatorname{Re}(z) \geq 0\}$ with maximum modulus

$$
M_{\Omega}(r, f)=\max \{|f(z)|: z \in \Omega,|z| \leq r\},
$$

and assume that $f(n)$ is an integer for all sufficiently large positive integers $n$. If $f$ satisfies (1) as $r \rightarrow \infty$ for some $N>0$, with $M(r, f)$ replaced by $M_{\Omega}(r, f)$, then again there exist polynomials $P_{1}$ and $P_{2}$ with $f(z) \equiv P_{1}(z) 2^{z}+P_{2}(z)$. Further, if $f$ takes integer values at all the non-negative integers and

$$
\limsup _{|z| \rightarrow \infty, z \in \Omega} \frac{|f(z)|}{2^{|z|}}<1
$$

then $f$ is a polynomial.

We remark that a result was proved in [22] for functions holomorphic on the product $\Omega^{n}$ of $n$ half-planes and taking integer values on $\mathbb{N}^{n}$. This result contains [8, Lemma 5], but not the theorem from [13]. We are very grateful to the referee for drawing our attention to this reference and to others such as $[1,2,23,24]$.

In order to state our result some terminology will be required. Let $f$ be analytic in $\Omega$, and let $0 \leq \lambda<\infty$. Then $f$ is of exponential type $\lambda$ in $\Omega$ if

$$
\limsup _{r \rightarrow \infty} \frac{\log ^{+} M_{\Omega}(r, f)}{r}=\lambda
$$

where $\log ^{+} x=\max \{0, \log x\}$ and $M_{\Omega}(r, f)$ is as in (2). This is of course in direct analogy with the definition of exponential type for entire functions. The main result to be proved is the following half-plane analogue of a theorem of Waldschmidt for entire functions [17].

Theorem 1.1. Let $d, J, \lambda$ satisfy

$$
0<d<1, \quad J \in \mathbb{N}, \quad \lambda>0, \quad 16\left(\frac{1+\log (1+J / 2)}{J}\right)+8(J-1) \lambda<d^{2} .
$$


Let $E \subset \mathbb{N}$ have lower linear density

$$
\underline{D}(E)=\liminf _{n \rightarrow \infty} \frac{|E \cap\{1, \ldots, n\}|}{n}>d,
$$

where $|X|$ denotes the number of elements of the set $X$. Let the function $f$ be analytic of exponential type less than $\lambda$ in the closed right half-plane $\Omega$, and assume that $f(n) \in \mathbb{Z}$ for every $n \in E$. Then $f$ is a polynomial.

\section{Lemmas used in the proof of Theorem 1.1}

\subsection{Linear forms}

The following lemma is a slight modification of [6, Lemma I, p.11]: a proof is given for completeness.

Lemma 2.1. Let $A \geq 1$ and $N \geq 2$ be integers. Suppose that $L_{1}, \ldots, L_{m}$ are linear forms in the $n$ variables $x_{1}, \ldots, x_{n}$, with real coefficients $a_{j, k}$ for $j=1, \ldots, m$ and $k=1, \ldots, n$, that $i s$,

$$
L_{j}=a_{j, 1} x_{1}+\ldots+a_{j, n} x_{n} .
$$

Suppose further that $n>m$ and

$$
\max _{j, k}\left|a_{j, k}\right| \leq A
$$

Then there exist integers $x_{1}, \ldots, x_{n}$, not all zero, such that

$$
\left|L_{j}\right| \leq \frac{1}{N}
$$

for $j=1, \ldots, m$, and

$$
\left|x_{k}\right| \leq 2 \cdot(2 n A N)^{\frac{m}{n-m}}
$$

for $k=1, \ldots, n$.

Proof. Define $X$ by

$$
X=\left[(2 n A N)^{\frac{m}{n-m}}\right],
$$

where $[x]$ denotes the greatest integer not exceeding $x$. An $n$-tuple of integers $\left(x_{1}, \ldots, x_{n}\right)$, in which each $x_{k}$ has absolute value no greater than $X$, 
gives rise to a point $\left(L_{1}, \ldots, L_{m}\right)$ lying in the closed $m$-dimensional cube of centre $(0, \ldots, 0)$ and side length $2 n A X$. Divide this cube into $(2 n A X N)^{m}$ closed subcubes each of side length $1 / N$. The number of distinct $n$-tuples $\left(x_{1}, \ldots, x_{n}\right)$ is evidently

$$
(2 X+1)^{n} \geq\left(2 \cdot(2 n A N)^{\frac{m}{n-m}}-1\right)^{n}>(2 n A N)^{\frac{n m}{n-m}} \geq(2 n A X N)^{m},
$$

since if this is not the case then we get

$$
(2 n A N)^{n}<(2 n A X N)^{n-m} \leq(2 n A N)^{n-m} \cdot(2 n A N)^{m},
$$

which is impossible. Hence there are distinct $n$-tuples giving rise to points $\left(L_{1}^{\prime}, \ldots, L_{m}^{\prime}\right)$ and $\left(L_{1}^{\prime \prime}, \ldots, L_{m}^{\prime \prime}\right)$ lying in the same subcube. But then we may write

$$
\left|\sum_{k=1}^{n} a_{j, k}\left(x_{k}^{\prime}-x_{k}^{\prime \prime}\right)\right|=\left|L_{j}^{\prime}-L_{j}^{\prime \prime}\right| \leq \frac{1}{N}
$$

for $j=1, \ldots, m$, where

$$
\left|x_{k}^{\prime}-x_{k}^{\prime \prime}\right| \leq 2 X \leq 2 \cdot(2 n A N)^{\frac{m}{n-m}}
$$

and $x_{k}=x_{k}^{\prime}-x_{k}^{\prime \prime} \neq 0$ for at least one $k$. This completes the proof.

\subsection{An application of the maximum principle}

Lemma 2.2. Let d, $M, L, K$ satisfy

$$
0<d<1, \quad M>0, \quad 1<K<L<\infty, \quad M L^{2} K<d^{2}(L-K) .
$$

Let $G \subseteq \mathbb{N}$ and let $F$ be analytic in the closed right half-plane $\Omega$ such that $F(z) \in \mathbb{Z}$ for all $z \in G$. Let $s>0$ be such that $M_{\Omega}(L s, F) \leq e^{M L s}$ and $F$ has $m \geq d s$ distinct zeros in $G \cap[1, s]$. Then $F(z)=0$ for all $z \in G \cap[s, K s]$.

Proof. Let $x_{1}, \ldots, x_{m}$ be distinct zeros of $F$ in $G \cap[1, s]$. For $0<x \leq s$ let

$$
p(z)=p(z, x)=\frac{z-x}{z+x} .
$$

Then $p$ satisfies

$$
|p(z)|=1 \quad(z \in i \mathbb{R}), \quad|p(z)| \geq \frac{L s-x}{L s+x} \quad(|z|=L s),
$$


and

$$
|p(z)| \leq \frac{K s-x}{K s+x} \quad(z \in[s, K s] \subseteq \mathbb{R}),
$$

the last estimate following from monotonicity. Next, let

$$
g(x)=\log \left[\left(\frac{L s+x}{L s-x}\right)\left(\frac{K s-x}{K s+x}\right)\right] .
$$

Then, for $0 \leq x \leq s$,

$$
\begin{aligned}
g^{\prime}(x) & =\frac{1}{L s+x}+\frac{1}{L s-x}-\frac{1}{K s+x}-\frac{1}{K s-x} \\
& =\frac{2 L s}{L^{2} s^{2}-x^{2}}-\frac{2 K s}{K^{2} s^{2}-x^{2}} \\
& =\frac{2 s^{3} K L(K-L)+2 x^{2} s(K-L)}{\left(L^{2} s^{2}-x^{2}\right)\left(K^{2} s^{2}-x^{2}\right)} \\
& \leq \frac{2(K-L)}{L K s}
\end{aligned}
$$

and hence

$$
g(x) \leq \frac{2 x(K-L)}{L K s} .
$$

The function

$$
F_{1}(z)=F(z) \prod_{j=1}^{m} \frac{1}{p\left(z, x_{j}\right)}
$$

is analytic in $\Omega$ and satisfies

$$
\left|F_{1}(z)\right| \leq M_{\Omega}(L s, F) \prod_{j=1}^{m} \frac{L s+x_{j}}{L s-x_{j}}
$$

on the boundary of the region given by $z \in \Omega,|z| \leq L s$, and this estimate also holds for $z \in[s, K s]$, by the maximum principle. For $z \in[s, K s]$ it 
therefore follows that

$$
\begin{aligned}
|F(z)| & \leq M_{\Omega}(L s, F) \prod_{j=1}^{m}\left[\left(\frac{L s+x_{j}}{L s-x_{j}}\right)\left(\frac{K s-x_{j}}{K s+x_{j}}\right)\right] \\
& =M_{\Omega}(L s, F) \exp \left(\sum_{j=1}^{m} g\left(x_{j}\right)\right) \\
& \leq M_{\Omega}(L s, F) \exp \left(\frac{2(K-L)}{L K s} \sum_{j=1}^{m} x_{j}\right) \\
& \leq M_{\Omega}(L s, F) \exp \left(\frac{2(K-L)}{L K s} \cdot \frac{m(m+1)}{2}\right) \\
& \leq \exp \left(M L s+\frac{d^{2}(K-L) s}{L K}\right)<1
\end{aligned}
$$

using (5) and the fact that the $x_{j}$ are distinct positive integers, which proves the lemma.

In order to apply Lemma 2.2, it is necessary for a given $d$ to choose $M$, $L$ and $K$ with (5) in mind. Evidently if

$$
M L^{2}<d^{2}(L-1)
$$

then $K$ may be chosen with $K-1$ small and positive so that (5) is satisfied. Since elementary calculus gives

$$
q(L)=\frac{L-1}{L^{2}} \leq q(2)=\frac{1}{4}
$$

for $1<L<\infty$, the appropriate condition is $4 M<d^{2}$.

Lemma 2.3. Let $0<d<1$ and $0<4 M<d^{2}$. Let $G \subseteq \mathbb{N}$ and let $F$ be analytic in $\Omega$ such that $F(z) \in \mathbb{Z}$ for all $z \in G$. Let $S>0$ be such that

$$
Q(r)=|G \cap[0, r]| \geq d r \quad \text { and } \quad M_{\Omega}(r, F) \leq e^{M r}
$$

for all $r \geq S$, and assume that $F(z)=0$ for all $z$ in $G \cap[1, S]$. Then $F(z)=0$ for all $z \in G$. 
Proof. Choose $L=2$ and $K \in(1,2)$ such that (5) is satisfied. Then $F$ has at least $d S$ distinct zeros in $G \cap[1, S]$. Applying Lemma 2.2 with $s=S$ then shows that $F(z)=0$ for all $z \in G \cap[S, K S]$, from which it follows at once that $F$ has at least $Q(K S) \geq d K S$ distinct zeros in $[1, K S]$. Hence Lemma 2.2 may again be applied, this time with $s=K S$. Repetition of this argument proves Lemma 2.3.

\subsection{The Nevanlinna characteristic in a half-plane}

This section provides a brief overview of a half-plane characteristic analogous to the Nevanlinna characteristic in the plane, the details of which may be found in $[7$, p.38]. Let $f$ be meromorphic in the closed upper half-plane

$$
\overline{\mathbb{H}}=\{z \in \mathbb{C}: \operatorname{Im}(z) \geq 0\}
$$

with poles at $\rho_{n} e^{i \psi_{n}}$, where $\rho_{n} \geq 0$ and $0 \leq \psi_{n} \leq \pi$. The counting function of the poles is

$$
c(r, f)=\sum_{1<\rho_{n} \leq r} \sin \psi_{n}
$$

and the integrated counting function takes the form

$$
C(r, f)=2 \int_{1}^{r} c(t, f)\left(\frac{1}{t^{2}}+\frac{1}{r^{2}}\right) d t=2 \sum_{1<\rho_{n} \leq r}\left(\frac{1}{\rho_{n}}-\frac{\rho_{n}}{r^{2}}\right) \sin \psi_{n} .
$$

The analogue of the Nevanlinna proximity function consists of the following two functions:

$$
\begin{aligned}
& A(r, f)=\frac{1}{\pi} \int_{1}^{r}\left(\frac{1}{t^{2}}-\frac{1}{r^{2}}\right)\left[\log ^{+}|f(t)|+\log ^{+}|f(-t)|\right] d t \\
& B(r, f)=\frac{2}{\pi r} \int_{0}^{\pi} \log ^{+}\left|f\left(r e^{i \phi}\right)\right| \sin \phi d \phi .
\end{aligned}
$$

The half-plane characteristic is then given by

$$
S(r, f)=A(r, f)+B(r, f)+C(r, f)
$$

and satisfies, for non-constant $f$ and $a \in \mathbb{C}$,

$$
S\left(r, \frac{1}{f-a}\right)=S(r, f)+O(1)
$$


as $r \rightarrow \infty$.

The following lemma uses the half-plane characteristic and is in the spirit of Carlson's theorem [5]. For generalisations in other directions see [23, 24].

Lemma 2.4. Let $E \subseteq i \mathbb{N}=\{i, 2 i, \ldots\}$ have lower density $D$. Let $f$ be analytic in $\overline{\mathbb{H}}$, of exponential type $\lambda<\pi D$, with $f(z)=0$ for all $z \in E$. Then $f \equiv 0$.

Here the lower density of $E$ and exponential type relative to the upper half-plane are defined in straightforward analogy with $\S 1$.

Proof. Assume that $f$ is not identically zero. As $r \rightarrow \infty$,

$$
B(r, f) \leq \frac{2}{\pi r} \int_{0}^{\pi}(\lambda+o(1)) r \sin \phi d \phi=O(1)
$$

and

$$
A(r, f) \leq \frac{1}{\pi} \int_{1}^{r}\left(\frac{1}{t^{2}}-\frac{1}{r^{2}}\right) 2(\lambda+o(1)) t d t+O(1) \leq \frac{2(\lambda+o(1))}{\pi} \log r .
$$

Since $f$ has no poles in $\overline{\mathbb{H}}$ applying (6) with $a=0$ now gives

$$
S(r, 1 / f) \leq A(r, f)+B(r, f)+O(1) \leq \frac{2(\lambda+o(1))}{\pi} \log r .
$$

But since the lower density of $E$ is $D$ we have

$$
c(r, 1 / f) \geq \sum_{n \in \mathbb{N} \cap(1, r], i n \in E} 1 \geq(D-o(1)) r
$$

as $r \rightarrow \infty$. Integrating this yields

$$
S(r, 1 / f) \geq C(r, 1 / f) \geq 2 \int_{1}^{r}(D-o(1)) t\left(\frac{1}{t^{2}}+\frac{1}{r^{2}}\right) d t \geq 2(D-o(1)) \log r
$$

as $r \rightarrow \infty$, which on combination with (7) gives $\lambda \geq \pi D$, a contradiction. Therefore $f$ must be identically zero. 


\subsection{A class of polynomials}

The following lemma summarises some basic facts from [17] concerning a class of polynomials which are key to the proof of Theorem 1.1.

Lemma 2.5. Define polynomials $p_{0}, p_{1}, \ldots$ by

$$
p_{0}(z)=1, \quad p_{1}(z)=z, \quad p_{h}(z)=\frac{z(z-1) \ldots(z-h+1)}{h !} \quad(h=2,3, \ldots) .
$$

Then $p_{h}(\mathbb{Z}) \subseteq \mathbb{Z}$ and for $R>0$ and $H \in \mathbb{N}$ we have

$$
\left|p_{h}(z)\right| \leq e^{H}\left(\frac{R}{H}+1\right)^{H} \quad \text { for }|z| \leq R, h=0, \ldots, H .
$$

Proof. It is easy to check that $p_{h}(\mathbb{Z}) \subseteq \mathbb{Z}$. To prove (9) write, following [17],

$$
\left|p_{h}(z)\right| \leq \frac{(R+H)^{h}}{h !} \leq \frac{H^{h}}{h !}\left(\frac{R}{H}+1\right)^{H} \leq e^{H}\left(\frac{R}{H}+1\right)^{H} .
$$

\subsection{Algebraic functions mapping integers to integers}

Proposition 2.6. Let the algebraic function $f$ be analytic in $\Omega$ and satisfy $f(E) \subseteq \mathbb{Z}$ for some set $E \subseteq \mathbb{N}$ of positive lower density. Then $f$ is a polynomial.

To prove Proposition 2.6, let $E$ and $f$ be as in the hypotheses, and assume that the lower density of $E$ exceeds $D>0$. We assert that $f$ maps the positive real axis into $\mathbb{R}$. To see this observe that the functions $\overline{f(\bar{z})}$ and $f(z)-\overline{f(\bar{z})}$ are algebraic because $f$ is. Since $f(z) \in \mathbb{R}$ for $z \in E$ and since an algebraic function having a sequence of zeros tending to infinity must vanish identically, the assertion follows.

Again since $f$ is algebraic there exists a positive integer $m$ such that, for all sufficiently large $r$,

$$
M_{\Omega}(r, f) \leq r^{m} .
$$

Let $n$ and $N$ be integers with $n / m$ and $N / n$ large, and in particular with

$$
D N \geq n+1 .
$$


Lemma 2.7. There exist arbitrarily large $r \in \mathbb{N}$ such that

$$
|E \cap\{r, r+1, \ldots, r+N-1\}| \geq n+1 .
$$

Proof. Assume that there exists $p_{0} \in \mathbb{N}$ such that, for every $p \geq p_{0}$,

$$
|E \cap\{N p, \ldots, N(p+1)-1\}| \leq n .
$$

Since the lower density of $E$ exceeds $D$ this gives, for large $p$,

$$
D N p \leq|E \cap\{1, \ldots, N p\}| \leq\left(p-p_{0}\right) n+O(1) \leq(n+o(1)) p,
$$

which contradicts (11).

Let $\varepsilon$ be small and positive and choose a large positive integer $r$ satisfying (12). Let $\Gamma=\Gamma_{r}$ be the circle of centre $r$, radius $\varepsilon r$, described once counterclockwise. Choose distinct

$$
a_{0}, \ldots, a_{n} \in E \cap\{r, r+1, \ldots, r+N-1\} .
$$

Then $a_{0}, \ldots, a_{n}$ lie inside $\Gamma$, since $r$ is large.

For $k=0, \ldots, n$ it follows from Cauchy's integral formula and the identity

$$
\frac{1}{t-z}=\frac{1}{t-a_{0}}+\frac{z-a_{0}}{\left(t-a_{0}\right)\left(t-a_{1}\right)}+\ldots+\frac{\left(z-a_{0}\right) \ldots\left(z-a_{k}\right)}{\left(t-a_{0}\right) \ldots\left(t-a_{k}\right)(t-z)},
$$

which is easily proved by induction, that

$$
f(z)=P_{k}(z)+\frac{1}{2 \pi i} \int_{\Gamma} \frac{\left(z-a_{0}\right) \ldots\left(z-a_{k}\right) f(t) d t}{\left(t-a_{0}\right) \ldots\left(t-a_{k}\right)(t-z)}
$$

for $z$ inside $\Gamma$, where

$$
P_{k}(z)=A_{0}+A_{1}\left(z-a_{0}\right)+\ldots+A_{k}\left(z-a_{0}\right) \ldots\left(z-a_{k-1}\right)
$$

is given by

$$
A_{j}=\frac{1}{2 \pi i} \int_{\Gamma} \frac{f(t) d t}{\left(t-a_{0}\right) \ldots\left(t-a_{j}\right)} .
$$

Thus $P_{k}(z)$ is the interpolating polynomial of degree at most $k$ which equals $f(z)$ at the $k+1$ points $a_{0}, \ldots, a_{k}[6$, p.103]. 
Next, let

$$
Q=\prod_{0 \leq j<k \leq n}\left|a_{k}-a_{j}\right| \leq C=N^{(n+1)^{2}},
$$

and observe that $C$ is independent of $r$. Since $f\left(a_{j}\right) \in \mathbb{Z}$ for $j=0, \ldots, n$ it follows from (16) and the residue theorem that

$$
Q A_{j} \in \mathbb{Z} \quad \text { for } j=0, \ldots, n \text {. }
$$

On the other hand since $r$ is large (13) gives

$$
\left|t-a_{j}\right| \geq \frac{\varepsilon r}{2}
$$

for $t \in \Gamma$. Thus combining (10), (16) and (17) yields for $m<j \leq n$, again since $r$ is large,

$$
\left|Q A_{j}\right| \leq \frac{C(\varepsilon r)(2 r)^{m}}{(\varepsilon r / 2)^{j+1}}<\frac{1}{2}
$$

which in conjunction with (18) gives $A_{j}=0$.

Recalling (14) and the definition (15) of $P_{k}$ it now follows that $P_{m}=P_{n}$ and that $f-P_{m}$ has $n+1$ zeros $a_{0}, \ldots, a_{n}$ in the interval $[r, r+N-1]$. Hence $f^{(n)}=\left(f-P_{m}\right)^{(n)}$ has a zero in the same interval, using Rolle's theorem. Since $r$ may be chosen arbitrarily large the algebraic function $f^{(n)}$ must vanish identically, and $f$ is a polynomial. This proves Proposition 2.6.

\section{Proof of Theorem 1.1}

Let $E \subseteq \mathbb{N}$ and $d, J, \lambda, f$ be as in the hypotheses. Label the elements of $E$ as $1 \leq \alpha_{1}<\alpha_{2}<\ldots$. Let $R$ be a large positive integer such that

$$
H=\frac{n}{J} \in \mathbb{N}, \quad \text { where } \quad m=|E \cap[1, R]| \text { and } n=2 m .
$$

Form the functions

$$
q_{\mu, \nu}(z)=p_{\mu}(z) f(z)^{\nu}, \quad \mu=0,1, \ldots, H-1, \quad \nu=0,1, \ldots, J-1,
$$

where $p_{\mu}$ is defined as in (8). This gives $H J=n$ functions which we label $g_{1}, \ldots, g_{n}$, where

$$
g_{k}(z)=p_{\mu(k)}(z) f(z)^{\nu(k)} .
$$


In order to prove Theorem 1.1, it suffices to show that the functions $g_{1}, \ldots, g_{n}$ are linearly dependent over $\mathbb{C}$. Once such a relation

$$
\sum_{k=1}^{n} B_{k} g_{k}(z) \equiv 0
$$

is established with the $B_{k}$ constants, not all zero, then it cannot be the case that there is an integer $q$ such that $B_{k} \neq 0$ implies $\nu(k)=q$, because $p_{h}$ has degree $h$ in (8). Hence it follows that $f$ is algebraic, and Proposition 2.6 shows that $f$ is a polynomial.

In order to prove that the $g_{k}$ are linearly dependent, observe first that

$$
a_{j, k}=g_{k}\left(\alpha_{j}\right) \in \mathbb{Z},
$$

using Lemma 2.5. Moreover, we have, for $j=1, \ldots, m$,

$$
\begin{aligned}
\left|a_{j, k}\right| & \leq e^{H}\left(\frac{R}{H}+1\right)^{H}\left(1+M_{\Omega}(R, f)\right)^{J-1} \\
& \leq e^{H}\left(\frac{R}{H}+1\right)^{H} e^{(J-1) \lambda R}=J(R) \leq A=[J(R)]+1,
\end{aligned}
$$

by (9), (20) and the fact that $R$ is large. Applying Lemma 2.1 with $N=2$ yields integers $A_{1}, \ldots, A_{n}$, not all zero, such that

$$
\sum_{k=1}^{n} A_{k} g_{k}\left(\alpha_{j}\right)=0
$$

for $j=1, \ldots, m$, and

$$
\left|A_{k}\right| \leq 8 n A
$$

since $n=2 m$. Set

$$
F(z)=\sum_{k=1}^{n} A_{k} g_{k}(z)
$$

Lemma 3.1. Choose a real number $M$ with

$$
4\left(\frac{1+\log (1+J / 2)}{J}\right)+2(J-1) \lambda<M<\frac{d^{2}}{4},
$$

using (4). Provided $R$ was chosen large enough we have

$$
|E \cap[1, r]| \geq d r \quad \text { and } \quad \log ^{+} M_{\Omega}(r, F) \leq M r \quad \text { for } \quad r \geq R .
$$


Proof. The first inequality of (26) holds provided $R$ was chosen large enough, since $E$ has lower density greater than $d$. Let $c$ denote positive constants which do not depend on $r$ or $R$. Then we have

$$
\begin{aligned}
M_{\Omega}(r, F) & \leq 8 n^{2} A e^{H}\left(\frac{r}{H}+1\right)^{H}\left(1+M_{\Omega}(r, f)\right)^{J-1} \\
& \leq c r^{2} e^{2 H}\left(\frac{r}{H}+1\right)^{2 H} e^{2(J-1) \lambda r}
\end{aligned}
$$

using (9), (21), (23) and the fact that $R$ is large. Now (19) gives

$$
\frac{r}{H} \geq \frac{R}{H}=\frac{R J}{n} \geq \frac{J}{2}
$$

Since the function

$$
\frac{1+\log (x+1)}{x}
$$

is decreasing for $x>0$ this yields, for $r \geq R$,

$$
\begin{aligned}
\log ^{+} M_{\Omega}(r, F) & \leq 2 r\left(\frac{H}{r}\right)\left(1+\log \left(\frac{r}{H}+1\right)\right)+2(J-1) \lambda r+O(\log r) \\
& \leq 4 r\left(\frac{1+\log (1+J / 2)}{J}\right)+2(J-1) \lambda r+O(\log r)<M r
\end{aligned}
$$

provided $R$ was chosen large enough.

The function $F$ satisfies $F(z) \in \mathbb{Z}$ for all $z \in E$, and $F(z)=0$ for all $z \in E \cap[1, R]$ by (22) and (24). It then follows from (25), (26) and Lemma 2.3 , with $S=R$ and $G=E$, that $F(z)=0$ for all $z \in E$. But (25) also gives

$$
4 M<d^{2}<d, \quad M<\pi d,
$$

and so (26) and Lemma 2.4, applied to the function $F(-i z)$, show that $F(z)$ vanishes identically, which completes the proof of Theorem 1.1.

\section{References}

[1] J.-P. Bézivin, Sur les points où une fonction analytique prend des valeurs entières, Ann. Inst. Fourier (Grenoble) 40 (1990), 785-809.

[2] J.-P. Bézivin, Suites d'entiers et fonctions entières arithmétiques, Ann. Fac. Sci. Toulouse Math. (6) 3 (1994), 313-334. 
[3] R.P. Boas Jr., Entire functions, (1954) Academic Press Inc., New York.

[4] R.C. Buck, Integral valued entire functions, Duke Math. J. 15 (1948), 879-891.

[5] W.H.J. Fuchs, A generalization of Carlson's theorem, J. London Math. Soc. 21 (1946), 106-110.

[6] A.O. Gelfond, Transcendental and Algebraic Numbers (1960) Dover Publications, New York.

[7] A.A. Gol'dberg and I.V.Ostrovskii, Distribution of values of meromorphic functions, (1970) Nauka, Moscow.

[8] J.K. Langley, On second order linear differential polynomials, Results Math. 26 (1994), 51-82.

[9] J.K. Langley, Two results related to a question of Hinkkanen, Kodai Math. J. 19 (1996), 52-61.

[10] J.K. Langley, Quasiconformal modifications and Bank-Laine functions, Arch. Math. (Basel) 71 (1998), 233-239.

[11] J.K. Langley, Integer points of meromorphic functions, Comput. Methods Funct. Theory 5 (2005), 253-262.

[12] J.K. Langley, Integer points of entire functions, Bulletin London Math. Soc. 38 (2006), 239-249.

[13] J.K. Langley, Integer-valued analytic functions in a half-plane, Comput. Methods Funct. Theory 7 (2007), 433-442.

[14] G. Pólya, Über ganze ganzwertige Funktionen, Nachrichten von der Gesellschaft der Wissenschaften zu Göttingen (1921), 1-10.

[15] Ch. Pommerenke, Boundary behaviour of conformal maps, (1992) Grundlehren der Mathematischen Wissenschaften [Fundamental Principles of Mathematical Sciences], 299. Springer-Verlag, Berlin.

[16] R.M. Robinson, Integer-valued entire functions, Trans. Amer. Math. Soc. 153 (1971), 451-468.

[17] M. Waldschmidt, Integer valued entire function on Cartesian products, Number Theory in Progress Vol. 1 (Zakopane-Koscielisko 1997), 553-576, de Gruyter, Berlin, 1999.

[18] R.V. Walliser, On entire functions assuming integer values in a geometric sequence, Theorie des Nombres (Quebec 1987), 981-989, de Gruyter, Berlin, 1989.

[19] M. Welter, Ensembles régulièrement lacunaires d'entier et fonctions entières arithmetiques, J. Number Theory 109 (2004), 163-181.

[20] M. Welter, A new class of integer-valued entire functions, J. reine Angew. Math. 583 (2005), 175-192. 
[21] J.M. Whittaker, Interpolatory function theory, (1935) Cambridge Tract No.33, Cambridge University Press.

[22] K. Yoshino, Pólya's theorem for nonentire functions, Rend. Circ. Mat. Palermo (2) (1984) Suppl. No. 3, 385-395.

[23] K. Yoshino, On Carlson's theorem for holomorphic functions, Algebraic Analysis Vol. II, 943-950, Academic Press, Boston MA, 1988.

[24] K. Yoshino, Arithmetic holomorphic functions of exponential type on the product of halfplanes, Tokyo Math. J. 18 (1995), no. 1, 147-150.

School of Mathematical Sciences, University of Nottingham, NG7 2RD. 